\title{
Chlorhexidine decontamination of sputum for culturing Mycobacterium tuberculosis
}

\author{
Shady Asmar ${ }^{1}$ and Michel Drancourt ${ }^{1,2^{*}}$
}

\begin{abstract}
Background: Culture of Mycobacterium tuberculosis is the gold standard method for the laboratory diagnosis of pulmonary tuberculosis, after effective decontamination.

Results: We evaluated squalamine and chlorhexidine to decontaminate sputum specimens for the culture of mycobacteria. Eight sputum specimens were artificially infected with $10^{5}$ colony-forming units (cfu)/mL Mycobacterium tuberculosis and Staphylococcus aureus, Pseudomonas aeruginosa and Candida albicans as contaminants. In the second step, we tested chlorhexidine-based decontamination on 191 clinical specimens, (Chlorhexidine, 0.1, 0.5 and 0.7 \%). In a last step, growth of contaminants and mycobacteria was measured in 75 consecutive sputum specimens using the routine NALC-NaOH decontamination protocol or with $0.7 \%$ chlorhexidine decontamination and an inoculation on Coletsos medium.

In the artificially model, contaminants grew in $100 \%$ of the artificially infected sputum specimens decontaminated using $100 \mathrm{mg} / \mathrm{mL}$ squalamine, in $62.5 \%$ of specimens decontaminated using N-Acetyl-L-Cysteine-Sodium Hydroxide (NALC-NaOH), and in $0 \%$ of specimens decontaminated using $0.1 \%, 0.35 \%$, or $1 \%$ chlorhexidine $(P<0.05)$. These specimens yielded $<10^{2} \mathrm{cfu} M$. tuberculosis using NALC-NaOH and $>1.4 .10^{2} \mathrm{cfu}$ M. tuberculosis when any concentration of chlorhexidine was used $(P<0.05)$.

In the second step we found that $0.7 \%$-chlorhexidine yielded $0 \%$ contamination rate, $3.2 \%$ for $0.5 \%$-chlorhexidine and $28.3 \%$ for $0.1 \%$-chlorhexidine. As for the 75 specimens treated in parallel by both methods we found that when using the standard NALC-NaOH decontamination method, 8/75 (10.7 \%) specimens yielded M. tuberculosis colonies with a time to detection of $17.5 \pm 3$ days and an $8 \%$ contamination rate. Additionally, 14 specimens yielded mycobacteria colonies (12 M. tuberculosis, and 2 Mycobacterium bolletii) (18.7\%) $(P=0.25)$, which has yielded a $100 \%$ sensitivity for the chlorhexidine protocol. Time to detection was of $15.86 \pm 4.7$ days $(P=0.39)$ and a $0 \%$ contamination rate $(P<0.05)$ using the $0.7 \%$-chlorhexidine protocol.
\end{abstract}

Conclusion: In our work we showed for the first time that chlorhexidine based decontamination is superior to the standard NALC-NaOH method in the isolation of $\mathrm{M}$. tuberculosis from sputum specimens. We currently use $0.7 \%$-chlorhexidine for the routine decontamination of sputum specimens for the isolation of M. tuberculosis and non-tuberculosis mycobacteria on egg-lecithin containing media.

Keywords: Mycobacterium tuberculosis, Decontamination, Chlorhexidine, Squalamine, N-acetyl-cysteine-sodium chloride, Culture, Contamination, Sensitivity, Standards for Reporting of Diagnostic Accuracy (STARD)

\footnotetext{
*Correspondence: michel.drancourt@univ-amu.fr

${ }^{1}$ Aix Marseille Université, URMITE, UM63, CNRS 7278, IRD 198, Inserm 1095,

Institut Hospitalo-Universitaire «Méditerranée Infection», AP-HM, 13005

Marseille, France

¿Unité de Recherche sur les Maladies Infectieuses et Tropicales Emergentes,

Faculté de Médecine, 27, Boulevard Jean Moulin, 13385 Marseille, Cedex 5,

France
}

\section{Biomed Central}

(c) 2015 Asmar and Drancourt. Open Access This article is distributed under the terms of the Creative Commons Attribution 4.0 International License (http://creativecommons.org/licenses/by/4.0/), which permits unrestricted use, distribution, and reproduction in any medium, provided you give appropriate credit to the original author(s) and the source, provide a link to the Creative Commons license, and indicate if changes were made. The Creative Commons Public Domain Dedication waiver (http://creativecommons.org/publicdomain/zero/1.0/) applies to the data made available in this article, unless otherwise stated. 


\section{Background}

The isolation and culture of Mycobacterium tuberculosis is the gold standard for laboratory pulmonary tuberculosis diagnoses, with a yet unsurpassed sensitivity of 100 colony-forming units per milliliter (cfu/mL) [1,2]. $M$. tuberculosis can be cultured from respiratory tract and stool specimens for pulmonary tuberculosis diagnoses $[3,4]$. In such specimens, the overgrowth of contaminants, including Staphylococcus aureus, Pseudomonas aeruginosa and Candida albicans, hampers the efficient isolation and culture of $M$. tuberculosis [5]. Various decontamination protocols have been proposed to limit the overgrowth of contaminants, in addition to adding antibiotics and antifungics to the culture media [6]. All of these protocols were shown to inhibit the viability of $M$. tuberculosis to some extent [7]. Sodium chloride $(\mathrm{NaOH})$-based protocols including the Petroff's method and the NALC-NaOH method, are widely used for decontamination $[8,9]$, despite the observation that $\mathrm{NaOH}$ can also affect $M$. tuberculosis viability; it was shown to kill up to $60 \%$ of $M$. tuberculosis bacilli in clinical specimens [7]. This may yield false negative results, especially in paucibacillary infection cases, such as those observed in HIV-infected patients [10].

Limited data have indicated that a chlorhexidine-based decontamination protocol, once proposed by our laboratory [11] and further used to decontaminate sputum [12,13] and stool specimens [4], performed better than some other methods for sputum specimen decontamination [11]. However, these studies were limited to the recovery of nontuberculosis mycobacteria. Chlorhexidine has not been thoroughly compared to the reference $\mathrm{NaOH}$ protocol for the laboratory diagnosis of pulmonary tuberculosis. Additionally, we recently observed that the broadspectrum, water-soluble cationic amino-sterol squalamine 1 (7,24-dihydroxylated-24 sulfated cholestane conjugated to spermidine group at C-3) [14] was ineffective against $M$. tuberculosis killing, with a minimum inhibitory concentration (MIC) $>100 \mathrm{mg} / \mathrm{L}$; therefore, it is a promising decontaminant [15]. Indeed, squalamine was revealed to have a high efficiency against gram-positive and gram-negative bacteria, with particular efficacy against $S$. aureus (MIC, $3.12 \mathrm{mg} / \mathrm{L}$ ) and $P$. aeruginosa (MIC, $8 \mathrm{mg} / \mathrm{L}$ ) as well as some activity against $C$. albicans $(\mathrm{MIC}>20 \mathrm{mg} / \mathrm{L} \mathrm{mg/L})$ [16]. Therefore, squalamine has the potential to be incorporated into decontamination protocols for the isolation of M. tuberculosis.

Here, we assessed various decontamination protocols by combining various concentrations of chlorhexidine combined with squalamine for the efficient culture of $M$. tuberculosis from sputum.

\section{Methods}

\section{Artificially infected sputum specimens}

Clinical isolates of $C$. albicans, $P$. aeruginosa and $S$. aureus were calibrated to a final concentration of $10^{7} \mathrm{cfu} / \mathrm{mL}$. Four
M. tuberculosis strains (H37Rv CIP 104475 and three clinical isolates) were cultured on Coletsos (bioMérieux, Craponne, France), and the colonies were suspended in Mycobacteria Growth Indicator Tubes [(MGIT) Becton Dickinson, Le Pont-de-Claix, France]. The suspensions were rigorously vortexed using $3-\mathrm{mm}$ sterile glass beads (Sigma-Aldrich, Saint-Quentin-Fallavier, France) and bypassed four times through a 25-G needle in order to disperse any clumped cells. The homogenized suspensions were then calibrated to $10^{7} \mathrm{cfu} / \mathrm{mL}$ by optical density at $580 \mathrm{~nm}$ (Cell Density Meter; Fisher Scientific, Illkirch, France). Smear-negative sputum specimens that were prospectively collected from our laboratory were mixed, sterilized by autoclaving at $121{ }^{\circ} \mathrm{C}$ for $15 \mathrm{~min}$ and centrifuged at $2500 \mathrm{~g}$ for $5 \mathrm{~min}$. The supernatants were inoculated with $P$. aeruginosa, $C$. albicans and $S$. aureus in addition to one of the four different $M$. tuberculosis suspensions in order to achieve a final concentration of $10^{5} \mathrm{cfu} / \mathrm{mL}$ for each microorganism.

\section{The decontamination procedures}

For the chlorhexidine or squalamine decontamination protocols, an equal volume of $0.1 \%$ dithiothreitol (SigmaAldrich) and sputum specimens were mixed together for $10 \mathrm{~min}$ in a $50-\mathrm{mL}$ conical tube. Then, a triple volume of chlorhexidine $(0.1,0.35$ or $1 \%$ ) (Chlorhexidine digluconate, Sigma-Aldrich) or squalamine solution were added to yield a $100 \mathrm{mg} / \mathrm{L}$ final concentration. The samples were vortexed and incubated for $15 \mathrm{~min}$ at room temperature with a continuous agitation. In the cases when inoculations were conducted on $5 \%$ sheep-blood Columbia agar (COS, bioMérieux), $10 \mathrm{~mL}$ of neutralizing solution [200 mL phosphate buffered saline (PBS), $0.6 \mathrm{~g}$ egg-lecithin and $2 \mathrm{~mL}$ Tween 80] was added to the chlorhexidine-decontaminated specimens in order to inactivate the chlorhexidine, and the mix was vortexed and incubated for $5 \mathrm{~min}$ at room temperature. Next, PBS was added up to $40-\mathrm{mL}$, the suspension was centrifuged at $1700 \mathrm{~g}$ for $15 \mathrm{~min}$, the supernatant was discarded, and the pellet was resuspended in $0.5 \mathrm{~mL}$ of sterile PBS, $\mathrm{pH}$ 6.8. For the squalaminechlorhexidine protocol, the squalamine was added first and mixed followed by incubation for $10 \mathrm{~min}$ at room temperature, and the chlorhexidine was then added. The reference N-Acetyl-L-Cysteine-Sodium Hydroxide (NALC$\mathrm{NaOH}$ ) method was applied as previously described [9].

A $100-\mu \mathrm{L}$ volume of specimen decontaminated by chlorhexidine at different concentrations $(0.1,0.35$ and $1 \%)$, squalamine- $100 \mu \mathrm{g} / \mathrm{mL}$ or chlorhexidine- $0.1 \%$-squalamine$100 \mu \mathrm{g} / \mathrm{mL}$ and NALC-NaOH and artificially infected specimens was inoculated in parallel in duplicate on COS with a non-inoculated negative control to measure the survival of the contaminants and in triplicate on a modified Middlebrook 7H10 medium (MOD4), which was previously described [17], with a non-inoculated negative control 
to compare the M. tuberculosis viability using these different protocols. For the contaminants, colonies were observed after 24 - and 48 -h incubation at $37^{\circ} \mathrm{C}$ under a $5 \%$ $\mathrm{CO}_{2}$ atmosphere, into an incubator. Contaminant identification was confirmed using matrix-assisted laser desorption ionization-time of flight mass spectrometry (MALDI-TOFMS), as previously described [18]. For the M. tuberculosis, microcolonies were detected on MOD4 by autofluorescence, as previously described [17].For each Petri-dish of the inoculated media, five random autofluorescence photos were taken on the fifth day of incubation in microaerophilic atmosphere at $37{ }^{\circ} \mathrm{C}$ in order to determine the mean number of microcolonies that developed per microscopic field (x 12.5 magnification).

Chlorhexidine decontamination of clinical sputum specimens The samples used in this study, were sent to the Mycobacteria Reference Laboratory (Institut Méditerranée Infection, Marseille, France) from January to March 2014. This study has received the agreement of the Ethics Committee of this Institut on February 19, 2007.

A total of 191 sputum specimens including six Ziehlpositive specimens collected in 97 patients suspected of pulmonary tuberculosis, were prospectively decontaminated by chlorhexidine to measure the contamination ratio and recovery of mycobacteria. Based on on-going results, a total of 53 specimens were decontaminated using $0.1 \%$ chlorhexidine, 55 specimens using $0.5 \%$-chlorhexidine, and 83 specimens using $0.7 \%$-chlorhexidine.

Furthermore, 75 other sputum specimens were evaluated for decontamination and recovery of mycobacteria by comparing the routine NALC- $\mathrm{NaOH}$ decontamination protocol and a $0.7 \%$-chlorhexidine decontamination. A $250 \mu \mathrm{L}$-volume of decontaminated specimen was inoculated in a Coletsos tube. The colonies were counted and identified with Ziehl-Neelsen staining and quantitative real-time polymerase chain reaction (qPCR), which targeted the internal transcribed spacer (ITS) by incorporation of a forward (5'-GGTGGGGTGTGGTGTTTGA G-3') and a reverse primer (5'-CACGTCCTTCATCGGC TCTC-3') as well as a $M$. tuberculosis probe (6FAMGCTAGCCGGCAGCGTATCCAT). In the cases when the qPCR failed, colony identification was accomplished by standard $16 \mathrm{~S}$ rRNA gene PCR sequencing, as previously described [19]. All these experimental procedures were done by one of us (SA) who has particular expertise in the field.

\section{Statistical analyses}

The Fisher exact test was used to assess the significance of the differences in the contamination and isolation rates. The Kruskal-Wallis test was used to assess any significant differences in the microcolony numbers.

\section{Results}

\section{The artificially infected sputum specimens}

Using the reference NALC-NaOH protocol, we observed $<7$ colonies for any of the contaminants in 5/8 (62.5\%) inoculated COS medium plates. These contaminants were identified as $C$. albicans and $P$. aeruginosa by MALDITOF-MS. Using squalamine, we observed an uncountable number of colonies, which were identified as $S$. aureus, $P$. aeruginosa and $C$. albicans in $8 / 8$ (100 \%) specimens $(P<0.05)$. Using $1 \%, 0.35 \%$, and $0.1 \%$ chlorhexidine and $0.1 \%$ - chlorhexidine $/ 100 \mathrm{mg} / \mathrm{L}$ - squalamine, we observed no growing contaminants $(0 \%)(P=0.026$, Fisher exact test). For the four $M$. tuberculosis strains, the mean microcolony number that was detected per field resulting from the different decontamination methods are presented in Table 1. There was a significant difference in the $M$. tuberculosis colony number between the different chlorhexidine-based protocols and the reference NALC$\mathrm{NaOH}$ method $(P<0.05)$. The difference in the M. tuberculosis colony number between the $0.1 \%$-chlorhexidine treatment and the other chlorhexidine concentrations was significant $(P<0.05)$. The difference between the $0.35 \%$ chlorhexidine and $1 \%$-chlorhexidine treatments as well as between the $0.1 \%$-chlorhexidine and $0.1 \%$-chlorhexidine/ $100 \mu \mathrm{g} / \mathrm{ml}$-squalamine treatments was not statistically significant $(P>0.05)$.

\section{The clinical specimens}

The rate of contamination was respectively $28.3 \%(15 / 53)$, $3.2 \%(2 / 55)$ and $0 \%$ for $0.1 \%$-chlorhexidine, $0.5 \%$-chlorhexidine $(P<0.05)$ and $0.7 \%$-chlorhexidine $(P<0.05)$, respectively. Contaminants were identified as $S$. aureus (2) and Streptococcus spp. (13). Specimens decontaminated by $0.1 \%$-chlorhexidine yielded three $M$. tuberculosis isolates, with a mean time to detection of $14.67 \pm 5.1$ days (9-19). Nine mycobacteria were cultured from specimens treated by $0.7 \%$-chlorhexidine, including $7 \mathrm{M}$. tuberculosis isolates and $2 M$. avium isolates, with a $23 \pm 4.6$ days (17-30) mean time to detection $(P<0.05)$.

As for the 75 sputum specimens treated in parallel by the reference method and $0.7 \%$-chlorhexidine, NALC-NaOH decontamination yielded eight $(10.7 \%)$ M. tuberculosis isolates, which grew on Coletsos medium, with a mean time to detection of $17.5 \pm 3$ days (15-23 days) and an $8 \%$ contamination rate. The contaminants were identified as Candida orthopsilosis (1), Candida glabrata (1), Streptococcus oralis (2), Hafnia alvei (1) and Raoultella ornithinolytica (1). Using a $0.7 \%$-chlorhexidine decontamination treatment, 14 mycobacteria $(12 \mathrm{M}$. tuberculosis and $2 \mathrm{Myco-}$ bacterium bolletii) $(18.7 \%)$ isolates were observed $(P=$ 0.25 ), with a $100 \%$ sensitivity for Mycobacterium isolation when the NALC-NaOH is considered as the reference method with a mean time to detection of $15.85 \pm 4.7$ days (8-25 days) $(P=0.39)$ for all mycobacteria and $17.33 \pm$ 
Table 1 Mean, median and standard deviation in the number of $M$. tuberculosis microcolonies, after decontamination of artificially infected sputum samples

\begin{tabular}{|c|c|c|c|}
\hline M. tuberculosis H37Rv CIP 104475 & Mean & Median & $\begin{array}{l}\text { Standard } \\
\text { variation }\end{array}$ \\
\hline chlorhexidine $-0.1 \%$ & 844.4 & 736.5 & 361 \\
\hline $\begin{array}{l}\text { chlorhexidine }-0.1 \% \text { - } \\
\text { squalamine } 100 \mu \mathrm{g} / \mathrm{mL}\end{array}$ & 1288 & 1396 & 576.3 \\
\hline chlorhexidine $-0.35 \%$ & 477.1 & 453 & 154.5 \\
\hline chlorhexidine $-1 \%$ & 665 & 630 & 207.6 \\
\hline $\mathrm{NALC}-\mathrm{NaOH}$ & 71.9 & 66 & 58.8 \\
\hline M. tuberculosis clinical isolate 1 & Mean & Median & $\begin{array}{l}\text { Standard } \\
\text { variation }\end{array}$ \\
\hline chlorhexidine-0.1 \% & 2493.1 & 2624.5 & 553.2 \\
\hline $\begin{array}{l}\text { chlorhexidine- } 0.1 \% \text { - } \\
\text { squalamine } 100 \mu \mathrm{g} / \mathrm{mL}\end{array}$ & 1774.5 & 1309 & 498.2 \\
\hline chlorhexidine $-0.35 \%$ & 1259.6 & 1238.5 & 393.1 \\
\hline chlorhexidine $-1 \%$ & 1426.6 & 1434 & 357.5 \\
\hline $\mathrm{NALC}-\mathrm{NaOH}$ & 81.7 & 73 & 37.3 \\
\hline M. tuberculosis clinical isolate 2 & Mean & Median & $\begin{array}{l}\text { Standard } \\
\text { variation }\end{array}$ \\
\hline chlorhexidine- $0.1 \%$ & 556.1 & 621 & 155.9 \\
\hline $\begin{array}{l}\text { chlorhexidine- } 0.1 \% \text { - } \\
\text { squalamine } 100 \mathrm{\mu g} / \mathrm{mL}\end{array}$ & 440.4 & 433 & 134.6 \\
\hline chlorhexidine $-0.35 \%$ & 237.9 & 202 & 127.4 \\
\hline chlorhexidine $-1 \%$ & 184.7 & 180 & 50.7 \\
\hline $\mathrm{NALC}-\mathrm{NaOH}$ & 4.4 & 4 & 2.83 \\
\hline M. tuberculosis clinical isolate 3 & Mean & Median & $\begin{array}{l}\text { Standard } \\
\text { variation }\end{array}$ \\
\hline chlorhexidine-0.1 \% & 288.1 & 284.5 & 87.8 \\
\hline $\begin{array}{l}\text { chlorhexidine- } 0.1 \% \text { - } \\
\text { squalamine } 100 \mu \mathrm{g} / \mathrm{mL}\end{array}$ & 124.4 & 136 & 26.1 \\
\hline chlorhexidine $-0.35 \%$ & 174.9 & 194 & 76.5 \\
\hline chlorhexidine $-1 \%$ & 140.7 & 142.5 & 25.5 \\
\hline NALC-NaOH & 4.9 & 3 & 3.7 \\
\hline
\end{tabular}

3.08 days (14-25 days) for the $M$. tuberculosis colonies ( $P$ $=0.9)$ and $0 \%$ contamination $(P<0.05)$. All of the $M$. tuberculosis isolates that were observed using the NALC$\mathrm{NaOH}$ decontamination method were also isolated with $0.7 \%$-chlorhexidine (Table 2). The number of $M$. tuberculosis colonies that were cultured using the $0.7 \%$-chlorhexidine decontamination method $(8,47,52,83,>100,>100$, $>100$ and $>100$ colonies) was higher than those grown after the NALC-NaOH treatment $(12,7,8,31,13,17,66$ and $>100$ colonies) (Table 3, Fig. 1).

\section{Discussion}

The routine NALC-NaOH decontamination method for sputum specimens does not prevent all contaminations, as a 2-5\% ratio of contamination is routinely observed [20], some works reported higher contamination rate on LJ (10$14 \%)$ [21-23]. Here, we used artificially contaminated sputum in a first step to assess the efficacy of decontamination. Accordingly, a $8 \%$ contamination rate may be due to the fact that we incorporated several specimens from the same cystic fibrosis patients (13.3\%) which are usually contaminated with resistant flora [24]. Additionally, this protocol is known to alter the viability of $M$. tuberculosis in decontaminated sputum, as observed in this study [12]. We therefore compared alternative decontamination protocols.

Based on its reported activity against contaminants [16] and its lack of activity against mycobacteria, including $M$. tuberculosis [15], squalamine was a potential candidate for decontamination. However, our investigation evaluating artificially infected sputum specimens indicated that $100 \mathrm{mg} / \mathrm{L}$ squalamine did not eliminate contaminant bacteria. This observation contrasts with the observation that only C. albicans resists to $100 \mathrm{mg} / \mathrm{mL}$ squalamine (not published data). This agree with a previously published report that showed a MIC of chlorhexidine of $>20 \mathrm{mg} / \mathrm{L}$ [16]. These data suggest that sputum may contain interfering factors that further limit the activity of squalamine against $P$. aeruginosa and S. aureus.

Chlorhexidine was more effective than the routine NALC-NaOH protocol in decontaminating artificially

Table 2 Yield of mycobacteria and contaminants in 75 sputum samples, decontaminated by 0.7 \%-chlorhexidine or NALC-NaOH

\begin{tabular}{|c|c|c|c|c|c|c|c|}
\hline & & \multicolumn{5}{|l|}{ NALC-NaOH } & \multirow[b]{2}{*}{ Total } \\
\hline & & $\begin{array}{l}\text { Positive } M \text {. } \\
\text { tuberculosis }\end{array}$ & $\begin{array}{l}\text { Positive other } \\
\text { mycobacteria }\end{array}$ & $\begin{array}{l}\text { Contaminated/ } \\
\text { Positive }\end{array}$ & $\begin{array}{l}\text { Contaminated/ } \\
\text { Negative }\end{array}$ & Negative & \\
\hline \multirow{6}{*}{$\begin{array}{l}\text { Chlorhexidine- } \\
0.7 \%\end{array}$} & Positive M. tuberculosis & 7 & 0 & 1 & 2 & 2 & 12 \\
\hline & $\begin{array}{l}\text { Positive other } \\
\text { mycobacteria }\end{array}$ & 0 & 0 & 0 & 0 & 2 & 2 \\
\hline & Contaminated/Positive & 0 & 0 & 0 & 0 & 0 & 0 \\
\hline & $\begin{array}{l}\text { Contaminated/ } \\
\text { Negative }\end{array}$ & 0 & 0 & 0 & 0 & 0 & 0 \\
\hline & Negative & 0 & 0 & 0 & 3 & 58 & 61 \\
\hline & Total & 7 & 0 & 1 & 5 & 62 & 75 \\
\hline
\end{tabular}


Table 3 Number of $M$. tuberculosis colonies for the eight positive cultures on Coletsos medium in both $0.7 \%$ chlorhexidine and NALC-NaOH decontamination

\begin{tabular}{lll}
\hline \multirow{2}{*}{ Specimen } & \multicolumn{2}{l}{ Number of M. tuberculosis colonies detected } \\
\cline { 2 - 3 } & $0.7 \%$ Chlorhexidine & NALC-NaOH \\
\hline 1 & 8 & 12 \\
2 & 47 & 7 \\
3 & 52 & 8 \\
4 & 83 & 31 \\
5 & $>100$ & 13 \\
6 & $>100$ & 17 (+contamination) \\
7 & $>100$ & 66 \\
8 & $>100$ & $>100$ \\
\hline
\end{tabular}

infected and clinical sputum specimens at a $0.1,0.35$ and $1 \%$ concentration in replicate. Moreover, it yielded 10-100 more $M$. tuberculosis colonies than the standard NALC$\mathrm{NaOH}$ method in triplicates, in agreement with previously published data regarding growth of non-tuberculosis mycobacteria on Löwenstein-Jensen medium [12,13].
Contrarily to the results obtained previously, we observed that a $0.1 \%$-chlorhexidine concentration and a $100 \mathrm{mg} / \mathrm{mL}$ squalamine are ineffective for the decontamination of sputum ( $28.3 \%$ contamination rate). Therefore, we increased the concentration of chlorhexidine and found that a $0.7 \%$-chlorhexidine was optimal for a routine use.

We therefore used an optimized $0.7 \%$ chlorhexidine decontamination method for clinical sputum specimens and observed that this protocol yielded a non-significant, nevertheless, higher mycobacteria isolate number than the routine NALC-NaOH decontamination method (14 versus 8 isolates in 75 sputum specimens). Moreover, a higher $M$. tuberculosis colony number was also observed, which is in agreement with the results obtained in the artificial model. These observations extended to $M$. tuberculosis and any sputum specimen, which is in line with observations previously made for non-tuberculosis mycobacteria isolated from cystic fibrosis sputum [12,13]. It must be emphasized that chlorhexidine, which is potentially toxic for mycobacteria, must be inactivated by incorporation with egg-lecithin in the culture medium.

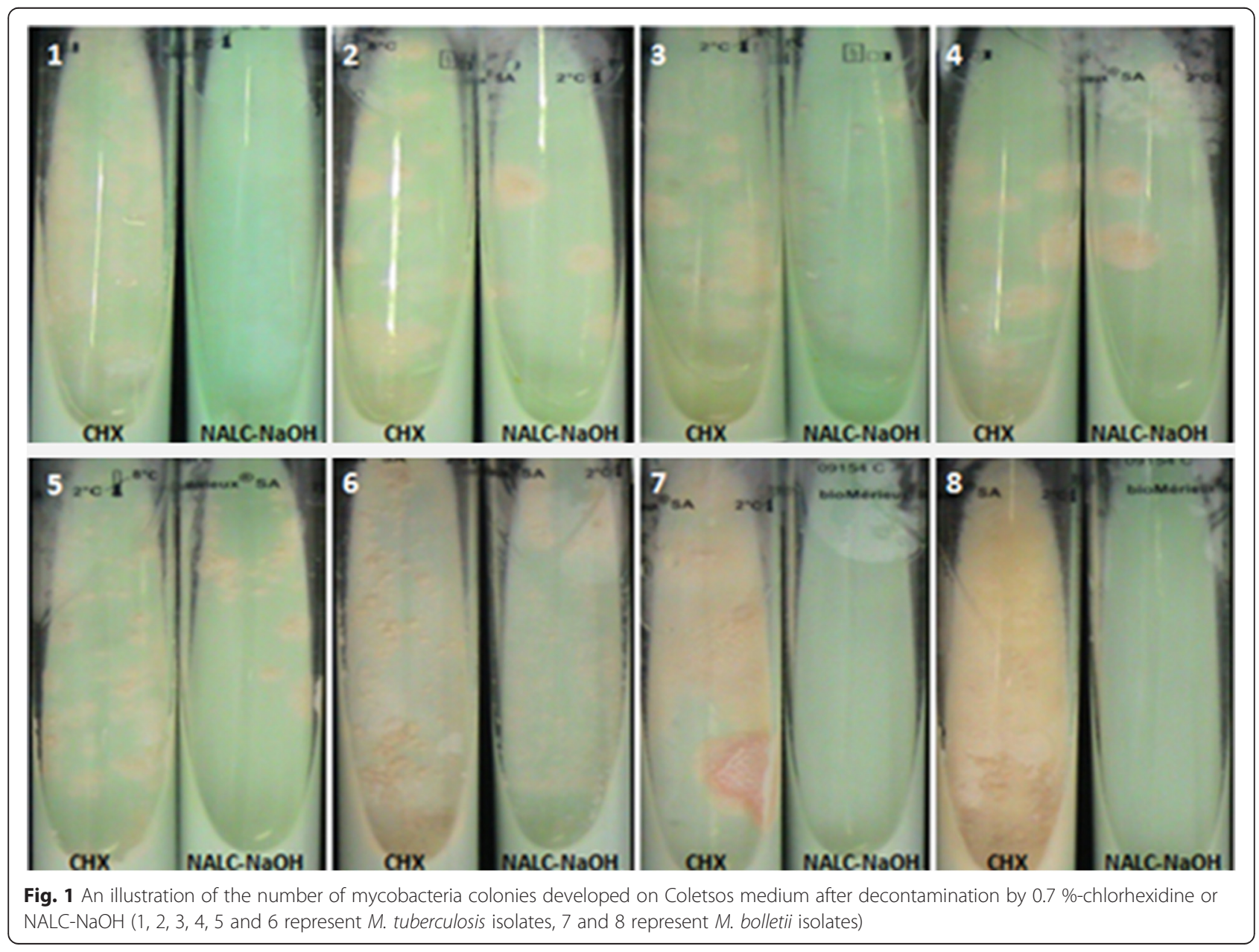




\section{Conclusions}

Here, we observed that a chlorhexidine-based decontamination method is effective against contaminants and more appropriate for the viability of mycobacteria. Moreover, we observed for the first time that routine chlorhexidinebased decontamination on specimens including those received from cystic fibrosis patients yielded superior results for the isolation, in particularly of M. tuberculosis than the standard NALC-NaOH decontamination method. At last, our preliminary cost evaluation based on 1000 specimens, indicates a $0.31 € /$ specimen cost of chlorhexidine lower than to the $1.4 € /$ specimen cost of reference NALC$\mathrm{NaOH}$ decontamination. These tabulations do not take into consideration the short 24-h expire delay for NALC$\mathrm{NaOH}$; when chlorhexidine can be stored for 3 months at room temperature.

We currently use a $0.7 \%$ chlorhexidine decontamination protocol combined with cultures on an egg-based medium [17] in our routine practice.

\section{Abbreviations}

CFU: Colony forming unit; NALC-NaOH: N-acetyl-L-cystein-sodium chloride; HIV: Human immunodeficiency virus; MIC: Minimum inhibitory concentration; MGIT: Mycobacteria growth indicator tube; MALDI-TOF-MS: Matrix-assisted laser desorption ionization-time of flight mass spectrometry; qPCR: Quantitative real-time polymerase chain reaction; ITS: Internal transcribed spacer; COS: Colombia agar $+5 \%$ sheep blood.

\section{Funding source}

Shady Asmar is supported by a PhD grant of IHU Méditerranée Infection.

\section{Competing interests}

The authors declare that they have no competing interests.

\section{Authors' contributions}

SA and MD contributed to study design. SA performed the lab experiments. SA and MD contributed to data interpretation and statistical design; SA performed the statistical analysis, prepared the first draft of the article with SA and MD contributing significant revision. SA and MD approved the final manuscript.

Received: 6 May 2015 Accepted: 7 July 2015

Published online: 05 August 2015

\section{References}

1. Alli OA, Ogbolu OD, Alaka OO. Direct molecular detection of Mycobacterium tuberculosis complex from clinical samples - An adjunct to cultural method of laboratory diagnosis of tuberculosis. N Am J Med Sci. 2011;3:281-8.

2. Tiruviluamala P, Reichman LB. Tuberculosis. Annu Rev Public Health. 2002;23:403-26.

3. Burdz TV, Wolfe J, Kabani A. Evaluation of sputum decontamination methods for Mycobacterium tuberculosis using viable colony counts and flow cytometry. Diagn Microbiol Infect Dis. 2003;47:503-9.

4. El Khéchine A, Henry M, Raoult D, Drancourt M. Detection of Mycobacterium tuberculosis complex organisms in the stools of patients with pulmonary tuberculosis. Microbiology. 2009;155:2384-9.

5. Kumar V, Balaji S, Gomathi NS, Venkatesan P, Sekar G, et al. Phage cocktail to control the exponential growth of normal flora in processed sputum specimens grown overnight in liquid medium for rapid TB diagnosis. J Microbiol Methods. 2006;68:536-42.

6. Kassaza K, Orikiriza P, Llosa A, Bazira J, Nyehangane D, et al. Lowenstein-Jensen selective medium for reducing contamination in Mycobacterium tuberculosis culture. J Clin Microbiol. 2014;52:2671-3.

7. Coulter P. Specimen Digestion, Decontamination for Mycobacteriology Culture Guidelines. 2009; Pro67-C-15-G.
8. Petroff SA. A new and rapid method for the isolation and cultivation of tubercle bacilli directly from the sputum and feces. J Exp Med. 1915;21:38-42.

9. Kubica GP, Dye WE, Cohn ML, Middlebrook G. Sputum digestion and decontamination with $\mathrm{N}$-acetyl-L-cysteine-sodium hydroxide for culture of mycobacteria. Am Rev Respir Dis. 1963;87:775-9.

10. Swai HF, Mugusi FM, Mbwambo KJ. Sputum smear negative pulmonary tuberculosis: sensitivity and specificity of diagnostic algorithm. BMC Res Notes. 2011;1:475.

11. Peres PJ, Gevaudan MJ, Gulian PC, de Micco P. Une méthode de traitement des produits pathologiques en vue de l'isolement des mycobactéries. Revue Fr Lab. 1988:173:67-74

12. De Bel A, De Geyter D, De Schutter I, Mouton C, Wellemans I, et al. Sampling and decontamination method for culture of nontuberculous mycobacteria in respiratory samples of cystic fibrosis patients. J Clin Microbiol. 2013;51:4204-6.

13. Ferroni $\mathrm{A}$, Vu-Thien $\mathrm{H}$, Lanotte $\mathrm{P}$, Le Bourgeois $\mathrm{M}$, Sermet-Gaudelus I, et al. Value of the chlorhexidine decontamination method for recovery of nontuberculous mycobacteria from sputum samples of patients with cystic fibrosis. J Clin Microbiol. 2006;44:2237-9.

14. Moore KS, Wehrli S, Roder H, Rogers M, Forrest Jr JN, et al. Squalamine: an aminosterol antibiotic from the shark. Proc Natl Acad Sci U S A. 1993;15(90):1354-8.

15. Ghodbane R, Ameen SM, Drancourt M, Brunel JM. In vitro antimicrobial activity of squalamine derivatives against mycobacteria. Tuberculosis (Edinb). 2013;93:565-6.

16. Salmi C, Loncle C, Vidal N, Letourneux Y, Fantini J, et al. Squalamine: an appropriate strategy against the emergence of multidrug resistant gram-negative bacteria? PLoS One. 2008;23(3), e2765.

17. Ghodbane R, Raoult D, Drancourt M. Dramatic reduction of culture time of Mycobacterium tuberculosis. Sci Rep. 2014;4:4236.

18. Seng P, Drancourt M, Gouriet F, Lascola B, Fournier PE, et al. Ongoing revolution in bacteriology: routine identification of bacteria by matrix-assisted laser desorption ionization time-of-flight mass spectrometry. Clin Infect Dis. 2009:49:543-51.

19. Drancourt M, Berger P, Raoult D. Systematic 165 rRNA gene sequencing of atypical clinical isolates identified 27 new bacterial species associated with humans. J Clin Microbiol. 2004;42:2197-202.

20. ATS. Diagnostic standards and classification of tuberculosis in adults children, 2000. Am J Respir Crit Care Med. 2000;161:1376-95.

21. Mukesh S, Rabindra NM, Nageswari RG, Savita VJ, Kalpana A, Vivian W. Comparison of modified Petroff's and N-acetyl-L-cysteine-sodium hydroxide methods for sputum decontamination in tertiary care hospital in India. Tuberculosis (Edinb). 2012;93:565-6.

22. Lee JJ, Suo J, Lin CB, Wang JD, Lin TY, Tsai YC. Comparative evaluation of the BACTEC MGIT 960 system with solid medium for isolation of mycobacteria. Int J Tuberc Lung Dis. 2003;7:569-74.

23. Samra Z, Kaufman L, Bechor J, Bahar J. Comparative study of three culture systems for optimal recovery of mycobacteria from different clinical specimens. Eur J Clin Microbiol Infect Dis. 2000;19:750-4.

24. Saiman L, Siegel JD, LiPuma JJ, Brown RF, Bryson EA, et al. Infection prevention and control guideline for cystic fibrosis: 2013 update. Infect Control Hosp Epidemiol. 2014;35 Suppl 1:S1-S67.

\section{Submit your next manuscript to BioMed Central and take full advantage of:}

- Convenient online submission

- Thorough peer review

- No space constraints or color figure charges

- Immediate publication on acceptance

- Inclusion in PubMed, CAS, Scopus and Google Scholar

- Research which is freely available for redistribution 